\title{
Interruption of Left Pulmonary Artery with Right Sided Aortic Arch with TOF: A Complete Anomaly Combination
}

\section{Kiran Gangadhar}

Department of Radio diagnosis and Imaging, IMS, Banaras Hindu University, Varanasi, India.

\begin{abstract}
Interruption of the pulmonary artery is a rare congenital disorder. The clinical symptoms are non-specific and radiological investigations are the key to diagnosis. We present a case of interruption of the left pulmonary artery associated with Tetralogy of Fallot in a young male. The radiological and clinical features of this condition are also reviewed.
\end{abstract}

Key words: Right sided aortic arch: Interruption of the pulmonary artery: Ventricular Septal Defect (VSD)

\section{Case History}

An 18-year-old male presented with an episode of hemoptysis and cough. He had a significant past history of recurrent chest infections and few episodes of hemoptysis. There was no history of tuberculosis in the past. On examination patient was mildly tachypneic (20 breaths per minute) with normal pulse rate and blood pressure. Chest examination revealed reduced breath sounds in left side with more laterally shifted apical pulse. Right side of chest revealed mild hyper resonant percussive noted with retrosternal mild hyper resonance.

Review of his chest radiographs (Figure A) showed a grossly enlarged heart (probably related to Tetralogy of Fallot), a right-sided

Correspondence to: Dr. Kiran Gangadhar Department of Radiodiagnosis and Imaging, Banaras Hindu University, Varanasi, India Email: kirang.585@googlemail.com aortic arch, and an oligaemic hypoplastic left lung. The right pulmonary artery and pulmonary trunk were enlarged, but the left hilum was indistinct. Rib notchings were seen at the left 4 th to 6 th ribs.

Computed tomographic (CT) pulmonary angiography was obtained during the patient's stay in the hospital revealed dilated right main pulmonary artery and its divisions (Figure B). There was, however, complete absence of the mediastinal portion of the left main pulmonary artery. Right sided aortic arch seen with mirror image branching with a large perimembranous VSD with dilated right ventricle suggestive of probable TOF. Prominent intercostals arteries and bronchial arteries were coursing within areas of extra pleural fat and mediastinum at several levels, supplying collateral circulation to the smaller left lung. Although the left lung was small, the bronchial branching pattern was normal with few patchy foci of pleural thickening. There was no evidence of bronchiectasis. Mild hyperinflation of the right lung was 
seen with transmediastinal herniation towards left.

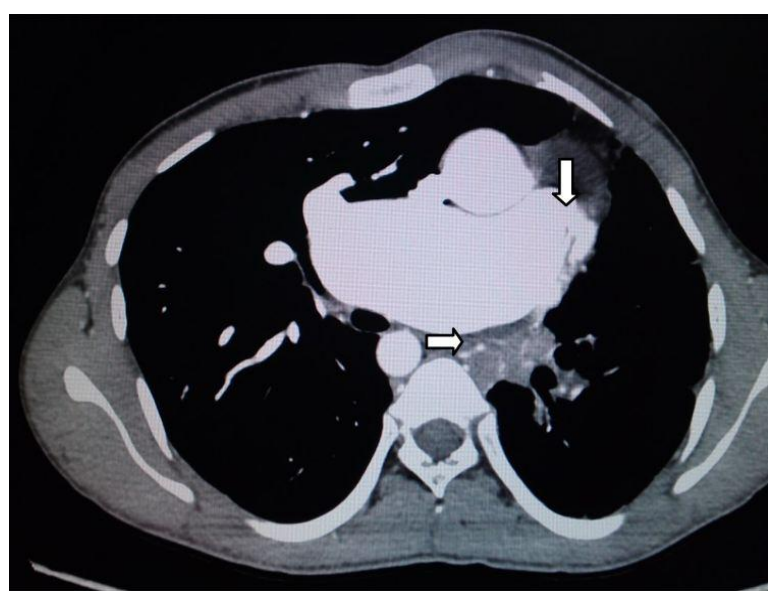

Figure A: axial CECT of lung with mediastinal window settings showing complete absence (vertical arrow) of the mediastinal portion of the left main pulmonary artery with dilated pulmonary trunk and right pulmonary main branch. Prominent mediastinum collateral (horizontal arrow) supplying circulation to the smaller left lung noted. Left lung was small however the bronchial branching pattern was normal with few patchy foci of pleural thickening. Hyperinflation of the right lung was seen with transmediastinal herniation towards left and asymmetric prominence of left chest wall.

\section{Discussion}

The main pulmonary arteries are derived from the proximal aspects of the sixth aortic arches during the first 16 weeks of intrauterine development ${ }^{1,}{ }^{16}$. Proximal interruption of the right or left pulmonary artery is an uncommon anomaly. It has been suggested that the term "interruption" be used in preference to "absence" of a pulmonary artery, in view of the fact that the intrapulmonary vascular network remains intact $^{2}$. With a blind end at the hilum, these pulmonary vessels continue to develop independently and receive oxygenated blood through systemic collateral vessels, such as the bronchial, intercostal, internal mammary, subclavian, or innominate arteries ${ }^{2,4}$. With interruption on the right, there may even be an anomalous artery arising from the ascending $\operatorname{aorta}^{3,4}$. In most cases, the interrupted pulmonary artery lies on the side

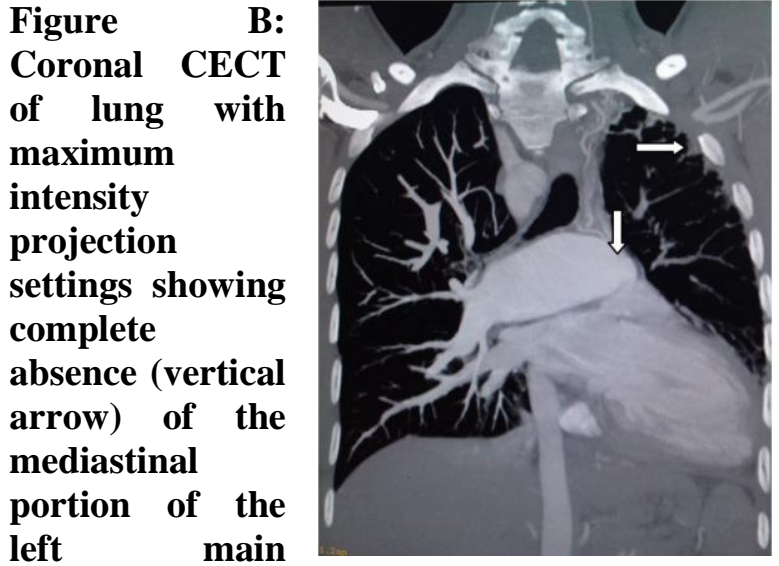

pulmonary artery with dilated pulmonary trunk and right pulmonary main branch. Prominent mediastinum collateral supplying circulation to the smaller left lung. Left lung was small, the bronchial branching pattern was normal with few patchy foci of pleural thickening (horizontal arrow).

opposite the aortic $\operatorname{arch}^{2}$. Interruption of the left pulmonary artery is usually associated with other cardiovascular congenital anomalies most commonly Tetralogy of Fallot

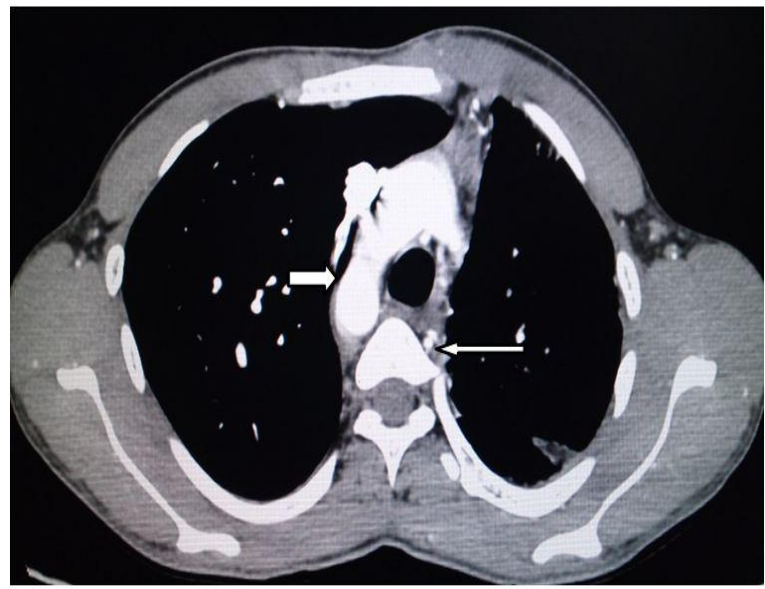

Fig C: axial CECT of lung with mediastinal window settings showing right side aortic arch (left headed horizontal arrow). Dilated pulmonary trunk and right pulmonary main branch. Prominent mediastinum collateral (right headed horizontal arrow) supplying circulation to the smaller left lung.

Patients with interruption of the right pulmonary artery have been grouped into three categories: group 1, those having an associated left-to-right shunt, usually a patent ductus arteriosus; group 2, those having associated pulmonary hypertension; and 
group 3, those having an isolated anomaly without pulmonary hypertension ${ }^{5}$. Where as individuals in groups 1 and 2 are unlikely to survive beyond infancy, those in group 3 often present as adults, either with incidental recognition of an abnormal chest radiograph or with hemoptysis.

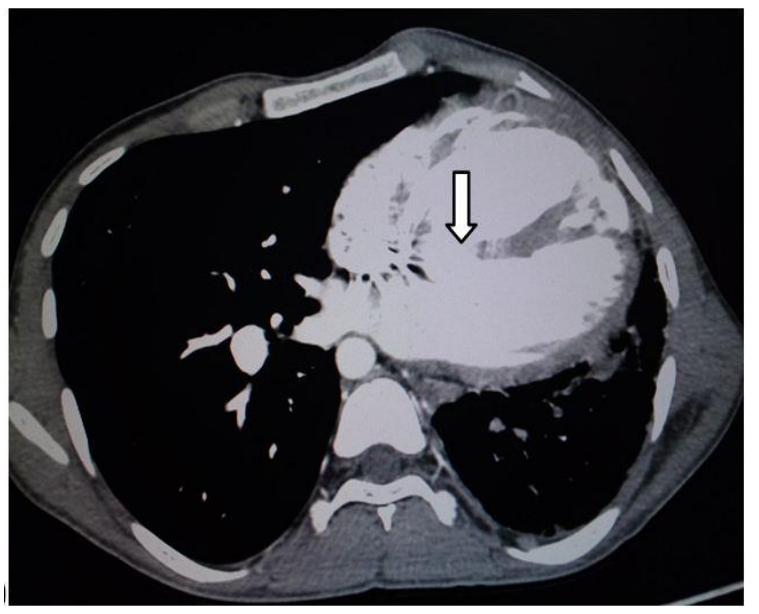

Fig D: axial CECT of lung with mediastinal window settings showing biventricular dilation with large perimembranous VSD suggestive of possible TOF.

Various radiological signs of an interrupted pulmonary artery can be detected on the plain chest radiograph. On the affected side, the hemi-thorax usually shows evidence of volume loss and the ipsilateral hilum is small and indistinct, because the corresponding pulmonary artery is interrupted. In wellestablished cases, rib notching may be visible due to collaterals from intercostal arteries. The unaffected hemi-thorax is often subject to compensatory hyperinflation of the lung, which may herniate into the affected side. The hilum of the unaffected side is enlarged, because it receives the entire output from right ventricle. These features are better appreciated in CT scans, which clearly show the abnormal vascular anatomy with intravenous contrast.

The interruption of the pulmonary artery can be revealed and collaterals supplying the affected lung are often visualised. The latter may be derived from bronchial, intercostal, internal mammary, subclavian or innominate arteries ${ }^{6}$. Secondary signs of collateral intercostal arteries such as rib notching and
Figure

Coronal volume rendering of $\mathbf{C T}$ pulmonary angiogram showing complete absence of the mediastinal portion of the left main pulmonary artery. Dilated pulmonary

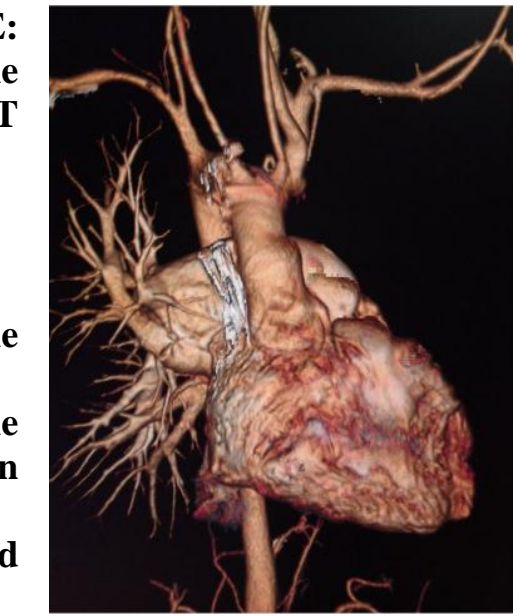
trunk and right pulmonary main branch. Right side aortic arch with mirror image branching noted.

serrated pleural thickening may be identified. The CT scan in the lung window is useful to confirm a normal bronchial branching pattern.

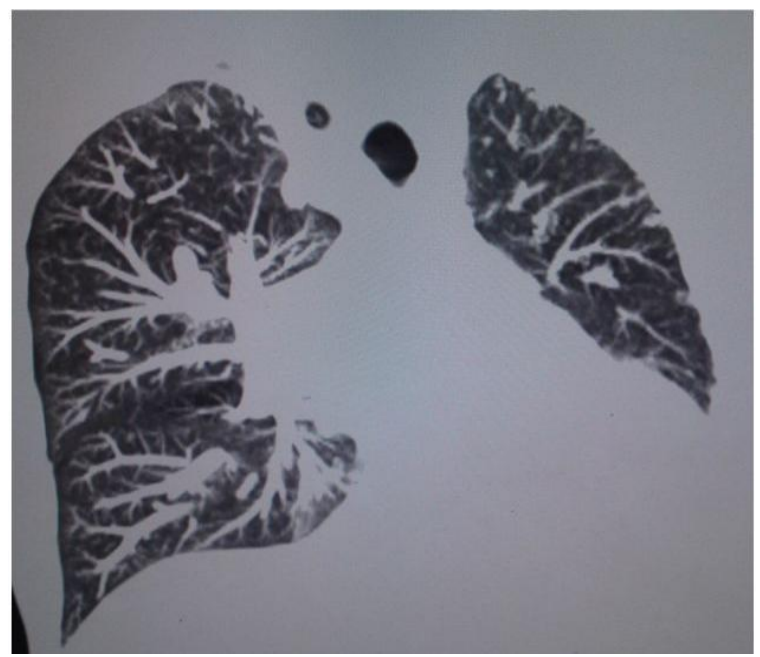

Figure F: coronal CECT of lung with lung window settings showing hyperinflation of the right lung with mildly reduced left lung size and peripheral pleural patchy nodularity. Grossly dilated right lung vasculature and right pulmonary artery noted.

Increased sub-pleural interstitial lung marking is another secondary sign of collateral intercostal arteries. Mosaic attenuation may be seen in both lungs, and is better revealed by high-resolution CT. The mosaic pattern in the affected lung may be related to hypoxic vasoconstriction and that in the unaffected lung to over-perfusion. 
Major radiological differential diagnoses of isolated interruption of the pulmonary artery include Swyer-James syndrome and hypogenetic lung syndrome. Swyer-James syndrome is related to childhood viral infection causing bronchiolitis and obliteration of small airways. While the affected lung is hyper lucent, the volume loss is less severe and there is evidence of airtrapping ${ }^{7}$.On the other hand, presence of scimitar sign, which represents an anomalous vein draining from right lung to below diaphragm, helps differentiate hypogenetic lung syndrome from interruption of pulmonary artery ${ }^{6}$.

There is still no consensus regarding the treatment of isolated interruption of the pulmonary artery. In asymptomatic patients, some clinicians adopt conservative management with close follow-up. Others opt for early revascularisation of the interrupted pulmonary artery to restore a physiological pulmonary circulation, which may lead to regression of pulmonary hypertension ${ }^{8,9}$. If massive haemoptysis ensues, selective embolization of systemic collaterals may be an option ${ }^{10}$.

\section{Conclusion}

In conclusion, interruption of pulmonary artery is a rare condition. Its clinical presentation is non-specifc and it is sometimes asymptomatic. Recognition of the relevant radiological features is the keystone of

diagnosis.

\section{References}

1. Grainger $\mathrm{R}$ G. The pulmonary circulation: the radiology of adaptation. Clin Radiol 1985; 36:103-116.

2. Kieffer SA, Amplatz K,Anderson RC, Lillehei CW. Proximal interruption of a pulmonary artery. Am J Roentgenenol Radium Ther Nucl Med 1963; 95:592597.
3. Fraser RG, Pare JAP, Pare PD, Fraser RS, Genereux GP. Diagnosis of diseases of the chest. Philadelphia, Pa: Saunders, 1988;729-731.

4. Sherrick DW, Kincaid OW, Du Shane JW. Agenesis of a main branch of th pulmonary artery. Am J Roentgenol Radium Ther Nucl Med 1962;87:917928.

5. Bahler RC, Carson P, Traks E, Levene A, Gillespie D. Absent right pulmonary artery: problems in diagnosis and management. Am J Med 1969;46:64-71

6. Davis SD. Case 28: Proximal interruption of the right pulmonary artery. Radiology. 2000; 217:437-40.

7. Bouros D, Pare P, Panagou P, Tsintiris $\mathrm{K}$, Siafakas N. The varied manifestation of pulmonary artery agenesis in adulthood. Chest. 1995;108:670-6.

8. Ten Harkel AD, Blom NA, Ottenkamp J. Isolated unilateral absence of a pulmonary artery: a case report and review of the literature. Chest. 2002;122:1471-7.

9. Welch K, Hanley F, Johnston T, Cailes $\mathrm{C}$, Shah MJ. Isolated unilateral absence of right proximal pulmonary artery: surgical repair and follow-up. Ann Thorac Surg. 2005;79:1399-402.

10. Reñé M, Sans J, Dominguez J, Sancho C, Valldeperas J. Unilateral pulmonary artery agenesis presenting with hemoptysis: treatment by embolization of systemic collaterals. Cardiovasc Intervent Radiol. 1995;18:251-4. 